\title{
Korkenzieher virtuell
}
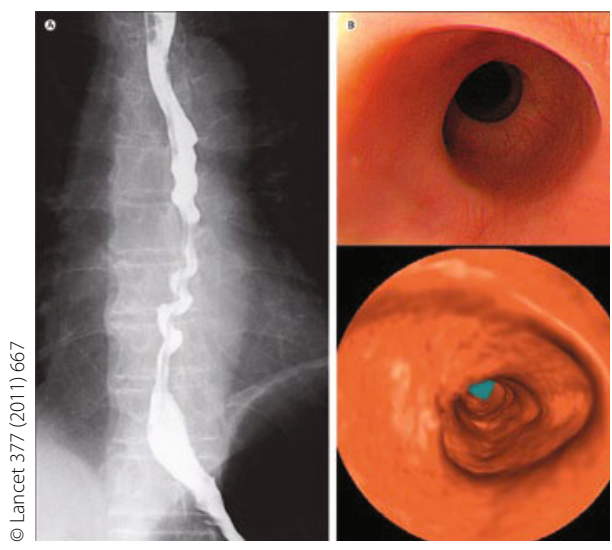

Korkenzieher-

ösophagus

im Barium-

breischluck (A)

und im

virtuellen CT (B).

Bei einem 52-jährigen beschwerdefreien Mann, der sich zum Routine-Check-up in einem Krankenhaus vorstellte, wurde eine Gastroskopie durchgeführt. Im Bereich des distalen Ösophagus erschien die Form des Lumens korkenzieherartig verändert. Ansonsten ergaben sich bei der Untersuchung keine Auffälligkeiten.

- Der Patient machte auch auf Befragen hin keine Angaben über Brustschmerzen oder eine Dysphagie. Ein Bariumbreischluck des Ösophagus (Abb. A) bestätigte den Verdacht auf eine wendeltreppenartige Peristaltik im Bereich des distalen Ösophagus und das Fehlen der Peristaltik im oberen Ösophagus. Bei einer virtuellen Endoskopie des Mediastinums konnte die korkzieherartige Konfiguration des Ösophagus dreidimensional in eindrucksvoller Weise dargestellt werden (Abbildung B). Obwohl für die Diagnose eines KorkenzieherÖsophagus eigentlich eine Manometrie erforderlich ist, wurde auf diese Untersuchung verzichtet, da der Patient beschwerdefrei und diese Diagnose bereits früher einmal gestellt worden war. Sollten sich Beschwerden einstellen, so wäre eine Therapie mit Muskelrelaxanzien und Anxiolytika indiziert. Bei manchen Patienten wird man eine antisekretorische Therapie mit Protonenpumpenhemmern oder sogar eine chirurgische Myotomie benötigen. Der Patient wurde mit dem Rat entlassen, keine kalten Getränke zu sich zu nehmen, da hiermit ein schmerzhafter Ösophagusspasmus ausgelöst werden könnte.

\section{Kommentar}

Die Bilder sind zwar ästhetisch eindrucksvoll, allerdings fragt man sich, warum bei einem beschwerdefreien Patienten ein ÖsophagusBreischluck und danach sogar ein virtuelles CT des Ösophagus angefertigt wurden. Vielleicht ja nur, um im medizinpublizistischen Blätterwald Aufmerksamkeit zu erregen.

- I. Hoshino et al.

(Department of Surgery, Numazu City Hospital, Higashi Sshij 550, Numazu, Shizuoka 410-0302, Japan): Corkscrew oesophagus on virtual endoscopy. Lancet 377 (2011) 667 